\title{
lodine-125 interstitial brachytherapy for malignant lacrimal sac tumours: an innovative technique
}

\author{
Ping Wang ${ }^{1} \cdot$ Nan $\mathrm{Ma}^{1} \cdot$ Shaobo Zhang ${ }^{1} \cdot$ Xiaona Ning $^{1} \cdot$ Chenjun Guo $^{1} \cdot$ Qiong Zhang $^{1} \cdot$ Qilin Cheng $^{1} \cdot$ \\ Yangjun Li $\mathbb{B}^{1}$
}

Received: 16 June 2020 / Revised: 2 July 2020 / Accepted: 9 July 2020 / Published online: 16 July 2020

(c) The Author(s), under exclusive licence to The Royal College of Ophthalmologists 2020

\begin{abstract}
Objectives Primary malignant tumours of the lacrimal sac are rare, surgery and radiotherapy may induce substantial side effects for patients. Here, this article reports an innovative technique of interstitial brachytherapy developed for the treatment of malignant lacrimal sac tumours.

Patients and methods Four patients (male 3, female 1), with an average age of 52.7 years (range 41-72 years), were individually diagnosed with squamous cell carcinoma, adenocarcinoma, adenoid cystic carcinoma and lymphoma. All patients received Iodine-125 interstitial brachytherapy after surgical resection for malignant lacrimal sac tumours. Visual function examination (vision, intraocular tension, fundus photography, fluorescein angiography, and optical coherence tomography) and CT/MRI/PET-MRI were performed to look for signs of recurrent tumours or metastasis.

Results Four patients were followed for an average of 28 months (range, 23-37 months). All patients were free from local disease. Their visual function was normal, and CT/MRI did not reveal any tumour recurrence.

Conclusions Iodine-125 interstitial brachytherapy can be used as an alternative to wide excision or exenteration of these tumours. There was good local control, reasonable maintenance of vision, and good cosmesis.
\end{abstract}

\section{Introduction}

Primary malignant tumours of the lacrimal sac, particularly of the nasolacrimal duct, are rare, thus generating a risk of late diagnosis and treatment in the clinic. Most tumours are primary and of epithelial origin (60-94\%), of which 55\% are malignant [1]. Lacrimal sac tumours typically present with epiphora and a palpable mass over the medial canthus and are thus often erroneously diagnosed as chronic dacryocystitis. Therefore, malignant lacrimal sac tumours can be potentially life-threatening due to local spread or metastatic disease, which emphasizes the relevance of early diagnosis and treatment [2].

These authors contributed equally: Ping Wang, Nan Ma

Yangjun Li

liyjun1969@126.com

1 Department of Ophthalmology, Tangdu Hospital, Fourth Military Medical University, Xi' an, Shaanxi, China
Surgery is the first choice of therapy for lacrimal sac tumours. If the mass extends into the orbit or nasolacrimal duct, orbital exenteration, lateral rhinotomy/paranasal sinus resection, and/or cervical lymph node dissection may be necessary [3]. These tumours not only cause loss of vision but also result in profound trauma for living patients. Postoperative tumours need to be treated with radiotherapy and chemotherapy. However, radiotherapy may induce radiation cataracts or glaucoma and lead to low vision, and chemotherapy as the treatment course demonstrated a marginal and nonsignificant improvement in overall survival and distant metastasis-free survival.

Interstitial brachytherapy, a new treatment, with the use of radioactive seeds (strontium-90, ruthenium-106 (Ru-106), or Iodine-125 (I-125)), has gained popularity in the treatment of certain intraocular and orbit tumours [3-7]. Sealy et al. was the first to use I-125 to treat ophthalmic tumours [8]. In 90 years ago, Moore has shown that tumours of the eyeball can be treated with a radioactive seed [5]. Some of these tumours include ciliochoroidal melanoma, retinoblastoma, Ewing sarcoma, squamous cell carcinoma, adenoid cystic carcinoma, and basal cell carcinoma 
$[3,4,6,7]$. In particular, I-125 is a common radioactive plaque used in this treatment. Other studies have used brachytherapy for certain orbital malignancies after orbital exenteration or enucleation [9-11]. However, there is no information available about the role of interstitial brachytherapy in the treatment of lacrimal sac tumours. We report our innovative technique and the effects on four patients with histopathologically confirmed malignant lacrimal sac tumours treated with radioactive I-125 seeds.

\section{Patients and methods}

\section{Patients}

This study was approved by the Ethics committee of the Tangdu Hospital at the Fourth Military Medical University (Xi'an, China) (No. 20170806). Informed consent and photographs were obtained from each patient. From 2017.4-2018.6, there were four patients with malignant lacrimal sac tumours in Tangdu Hospital. The histologic diagnoses were as follows: squamous cell carcinoma, adenocarcinoma, adenoid cystic carcinoma and B-cell nonHodgkin's lymphoma.

\section{Materials}

The I-125 radioactive particles were purchased from Tianjin Saide Medicine Co. Ltd. (Tianjin, China). The I-125 seed source was a sealed source for radionuclides. The parameters for the I-125 seed were as follows: activity $0.5-0.6 \mathrm{mCi}$; volume $4.5 \mathrm{~mm} \times \varnothing 0.8 \mathrm{~mm}$ (cylinder); energy $27-35 \mathrm{keV} \gamma$ source; half-life 59.6 days; tissue penetration $1.7 \mathrm{~cm}$; half-value layer $0.025 \mathrm{~mm}$ lead; source radiation activity $\sim 11.1-37.9 \mathrm{MBq}$; and the seed surface was covered with titanium alloy.

All I-125 seeds were placed in polythene tubes (PICC: peripherally inserted central catheter) to compose a strip. The numbers implanted depended on the tumour location and size. The ends of the tubes were sealed so that the I-125 seeds in the tubes did not move and they could be dislodged in the future if necessary.

\section{Surgical treatments}

All patients underwent haematologic examination, and orbital computed tomography (CT) and magnetic resonance imaging (MRI) were performed before the operation. Complete ultrasonography of the neck and abdominal lymph nodes ruled out the involvement of multiple organs (pancreas, kidneys, lymph nodes, retroperitoneum, etc.). The surgery was performed with standard general anaesthesia. The lacrimal sac, nasolacrimal canal and partial nasal mucosa were excised completely during the operation. The strip was implanted into the nasolacrimal canal or/and under the orbital periosteum. The number of seeds was calculated using the following formula: seed number $=$ (tumour length + width + height) $/ 3 \times 5 / 0.5$ (or 0.6 ) $\mathrm{mCi}$. The first and third patients underwent a tumour resection combined with I-125 seed implantation, while the frozen section assessment confirmed that the tumour was malignant during the operation. None of the patients received any other treatment.

Treated patients were followed with periodic ocular examination (fundus photography, fundus fluorescein angiography (FFA), optical coherence tomography (OCT)) and CT/MRI/positron emission tomography-MRI (PETMRI) to look for signs of recurrent tumour at every followup visit.

Patient characteristics before and after the operation are summarized in Tables 1 and 2.

\section{Results}

The median follow-up from the time of I-125 seed implantation for the four patients was 28 months (range, 23-37 months) until 2020.05. At 1, 3, 6, 9, and 12 months after the operation and then every 6 months thereafter, visual function examination and CT/MRI/PET-MRI were performed. The first follow-up was performed 1 month post-interstitial brachytherapy. CT and MRI scans of the orbit were performed at follow-up to ensure the correct location of the I-125 seeds, and the scans showed complete response with no evidence of disease.

\section{Patient 1}

The oldest female had the longest follow-up time. She had undergone gross tumour resection of a primary nasal cavity mass with no evidence of metastatic disease 5 years ago. After dacryocystectomy and I-125 seed implantation, the patient was reviewed every month and examined by $\mathrm{CT}$ and/or MRI. The follow-up time lasted for 37 months. Vision in her right eye was $80 / 100$. Coronal CT scans showed that the I-125 seeds in situ. The MRI showed no recurrence of the tumour, and the FFA and fundus photography were normal. Abdominal ultrasonography and thoracic X-ray showed no metastasis (Fig. 1).

\section{Patient 2}

The appearance of this male was normal 29 months after interstitial brachytherapy. During the 1 st month, the right eye demonstrated radiotherapy-related dermatitis. The eyelid skin was red and slightly painful. The patient 
Table 1 Individual case data.

\begin{tabular}{llllllll}
\hline ID & Age at Dx, years & Sex & R/L & Operation history & Path & Vision & Fundus photography \\
\hline 1 & 72 & F & R & Nasal tumour operation 5 years ago & Squamous cell carcinoma & 0.8 & Normal \\
2 & 41 & M & R & No & No & 1.0 & Normal \\
3 & 49 & M & L & DCR 1 month ago & Adenoid cystic carcinoma & 1.0 & Normal \\
4 & 49 & M & R & No & No & 1.0 & Normal \\
\hline
\end{tabular}

$D x$ diagnosis, $F$ female, $M$ male, Path pathologic diagnosis, $D C R$ dacryocystorhinostomy.

Table 2 Individual case data after operation.

\begin{tabular}{|c|c|c|c|c|c|c|c|c|}
\hline ID & Path & EOR & $\begin{array}{l}\text { TTF from } \\
\text { implant (month) }\end{array}$ & TNM & No. & Strength $(\mathrm{mCi})$ & Total BT dose Gy & Complications \\
\hline 1 & Squamous cell carcinoma & GTR & 37 & $\mathrm{~T}_{3} \mathrm{~N}_{0} \mathrm{M}_{0}$ & 10 & 0.5 & 89 & No \\
\hline 2 & Adenocarcinoma & GTR & 29 & $\mathrm{~T}_{1} \mathrm{~N}_{0} \mathrm{M}_{0}$ & 21 & 0.6 & 186 & Dermatitis \\
\hline 3 & Adenoid cystic carcinoma & GTR & 23 & $\mathrm{~T}_{2} \mathrm{~N}_{0} \mathrm{M}_{0}$ & 18 & 0.5 & 126 & No \\
\hline 4 & B-cell non-Hodgkin's lymphoma & GTR & 23 & $\mathrm{~T}_{2} \mathrm{~N}_{0} \mathrm{M}_{0}$ & 16 & 0.6 & 136 & No \\
\hline
\end{tabular}

Path pathologic diagnosis, EOR extent of resection, GTR gross total resection, TTF time to follow-up, BT brachytherapy.

Fig. 1 Pre- and post-operative images of the patient 1 . (1) The pictures of patient 1 before operation a and the dissected mass b. MRI showed midintensity on both T1-weighted (c, axial) and T2-weighted MRI (d, coronal) (yellow arrow: the tumour). (2) The picture of patient 1 after operation at 37 months a, and the vision was 0.8. Coronal CT scans showed that the I-125 seeds were stable b, and the MRI showed no tumour recurrence $\mathbf{c}$ (yellow arrow: the I-125 seeds trips). The FFA $\mathbf{d}$ and fundus photography e results were normal.
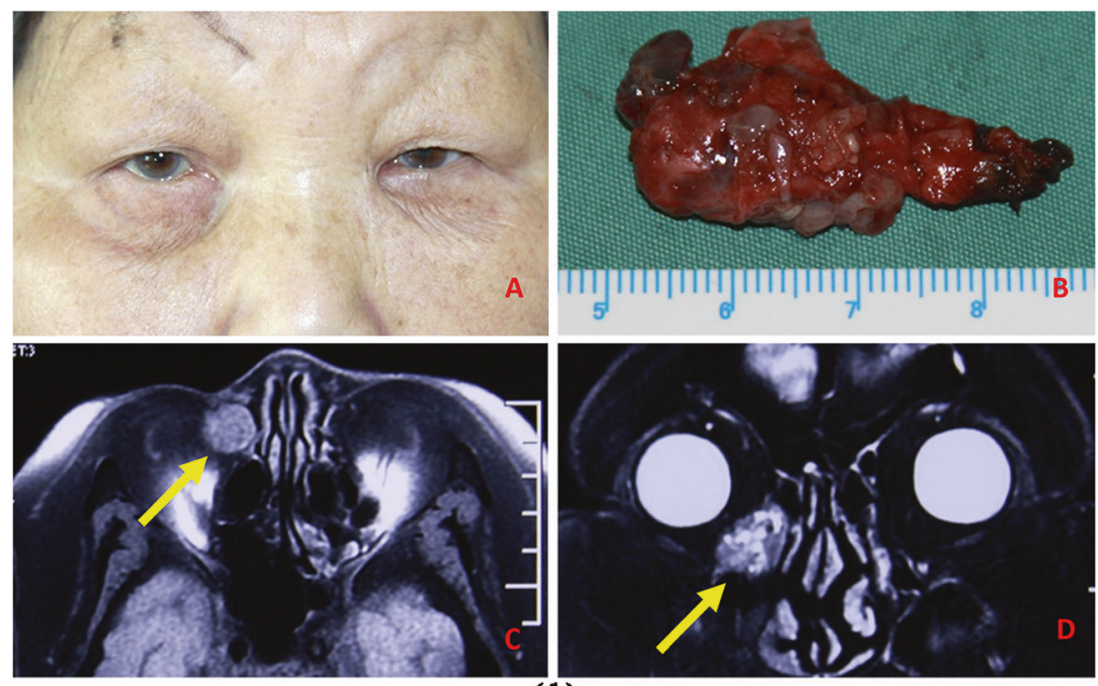

(1)

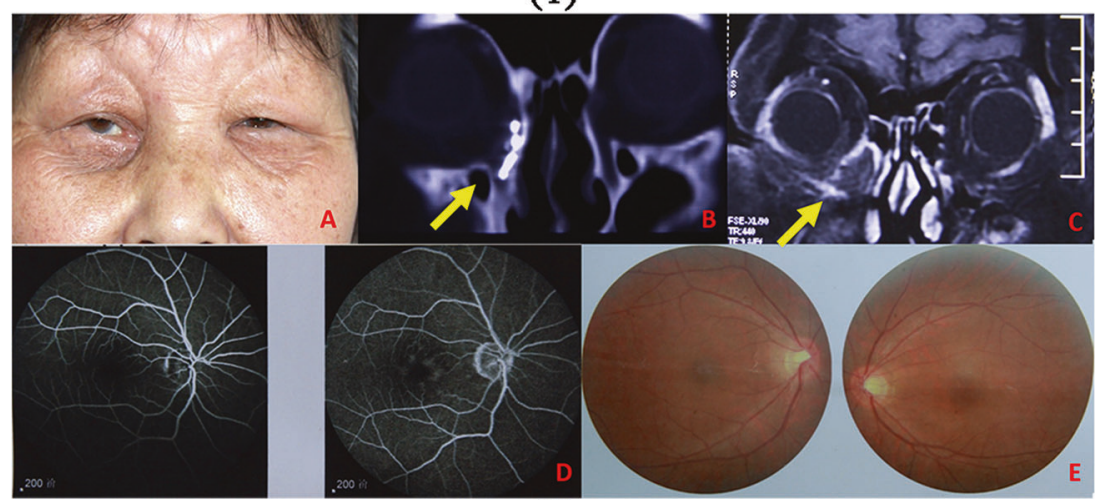

(2)

recovered after one month of treatment with an externally applied agent. CT showed that the I-125 seeds were in the nasolacrimal canal and under the orbital periosteum.
Vision in the right eye was 100/100, and the fundus photography results were normal. MRI showed no tumour growth (Fig. 2). 
Fig. 2 Pre- and post-operative images of the patient 2. (1) Clinical examination revealed a firm, localized, nontender mass at the inner canthus consistent with a lacrimal sac swelling a. The picture of the dissected mass b. Corresponding axial and coronal gadolinium-enhanced T1-weighted MRI scans showed high intensity c, $\mathbf{d}$ (yellow arrow: the tumour). (2) The picture of patient 2, 29 months after brachytherapy a. The CT scan showed that the I-125 seeds were in the nasolacrimal canal b under the orbital periosteum c (yellow arrow: the I-125 seeds trips). Vision in the right eye was 1.0 , and the fundus photography $\mathbf{d}$ result was normal. MRI showed no tumour growth $\mathbf{e}, \mathbf{f}$.

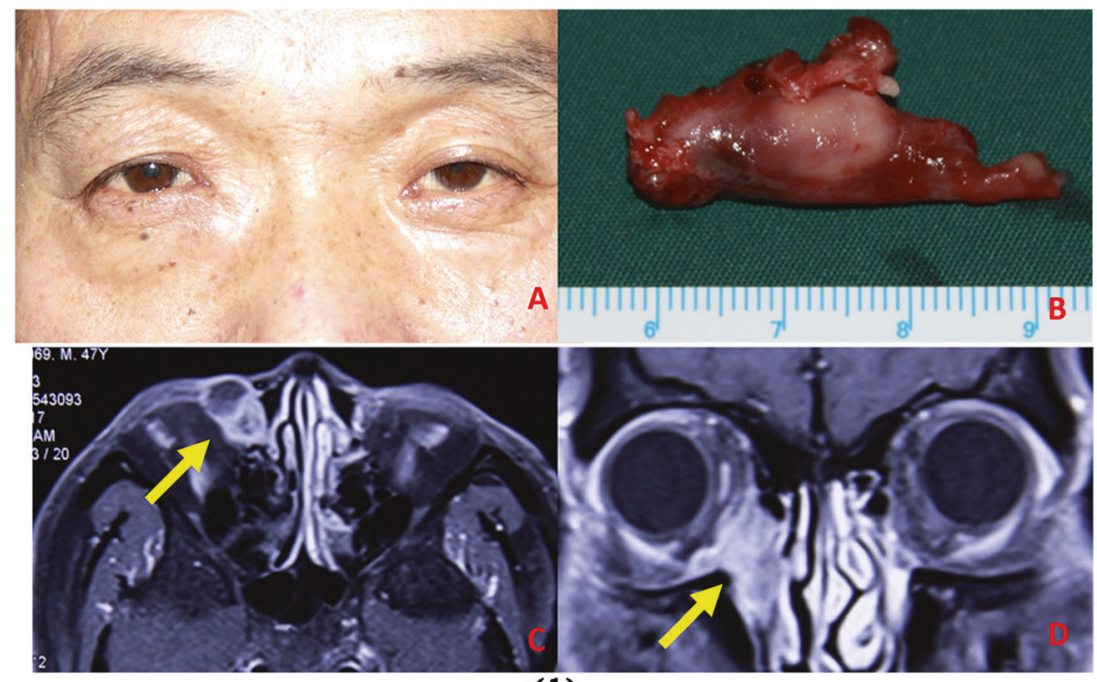

(1)
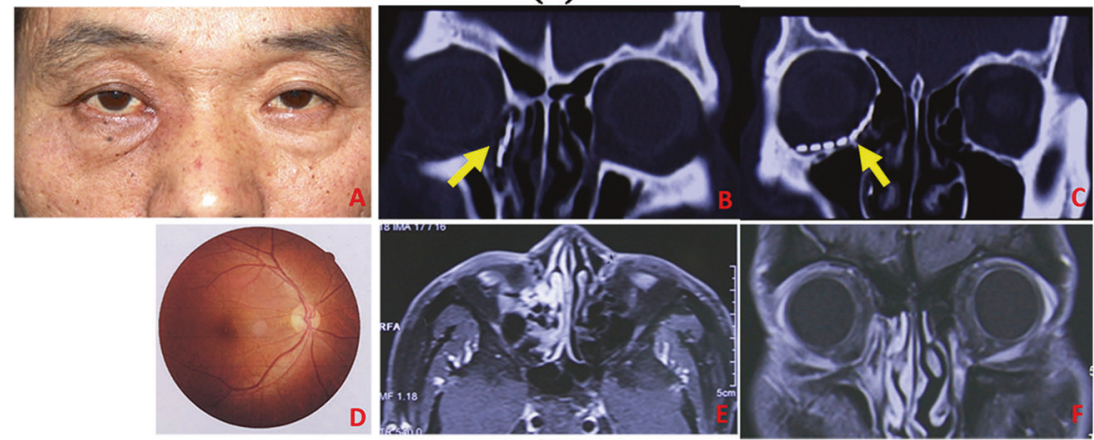

(2)

\section{Patient 3}

This male had a DCR operation in another hospital with residual tumour. Then he underwent PET-MRI before interstitial brachytherapy to make sure no metastasis. T1and gadolinium-enhanced T1-weighted MRI showed midintensity. One month after I-125 seeds implantation, the coronal CT scans showed that the I-125 seeds in situ and that there was no tumour recurrence. Visual function test, FFA, OCT and fundus photography results were normal. The lymph nodes in the neck did not demonstrate swelling on ultrasonography (Fig. 3).

\section{Patient 4}

The final pathologic diagnosis was B-cell non-Hodgkin's lymphoma. The patient underwent PET-MRI 1 month after interstitial brachytherapy, and the result was negative and showed that the patient was free from local disease. CT showed that the I-125 seeds in situ and that there was no tumour growth. The fundus photography and FFA results were normal, and vision was 100/100 (Fig. 4).

All patients have completed all treatment, are currently on regular follow-up regimens, and have excellent cosmetic and functional outcomes without any restriction of ocular mobility or vision loss. All patients experienced lacrimation, and the third patient experienced dryness of the eye. However, all vision, fundus photography, and ocular mobility were normal, and the tumour was not recurrent until now. All of the patients are under our follow-up care.

\section{Discussion}

Malignant lacrimal sac tumours are rare. Surgery is the first choice of treatment. In order to prevent tumour recurrence, brachytherapy or chemotherapy were needed in the later stage. Chemotherapy may cause many adverse effects, partially or throughout the body. Patients need to be carefully counselled about the possible postsurgical scarring and disfigurement cause by these treatments, along with the risks of toxicity from chemotherapy.

External radiotherapy can produce several direct and indirect effects on ocular and periocular structures, and it can lead to substantial visual loss, including cataracts, glaucoma, radiation retinopathy, dry eyes, and keratopathy [12-17]. The incidence of radiation retinopathy varies widely in the literature, depending on the irradiation 
Fig. 3 Pre-operative and postoperative images of the patient 3. (1) The picture of patient 3 after DCR in another hospital a. Axial CT scans showed a soft tissue mass in the left nasolacrimal fossa $\mathbf{b}$. The PETMRI showed high-saccharide metabolism of the lacrimal sac tumour $\mathbf{c}-\mathbf{f}$ (yellow arrow: the tumour). (2) The picture of patient 3, 23 months after brachytherapy a. Coronal CT scans showed that the I-125 seeds were stable and that there was no tumour recurrence b, c (yellow arrow: the I- 125 seeds trips). The FFA $\mathbf{d}$ and fundus photography e results were normal.
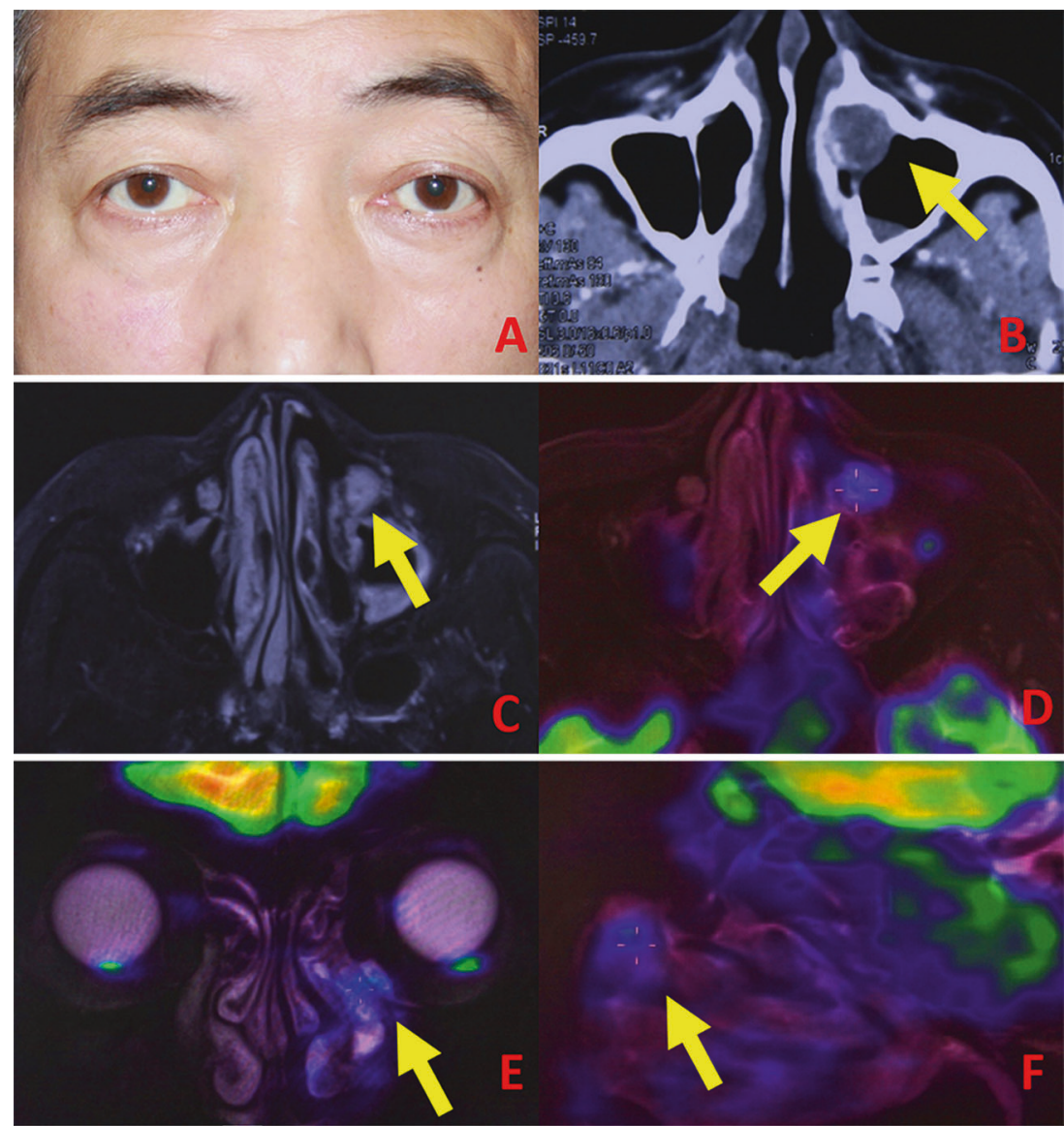

(1)

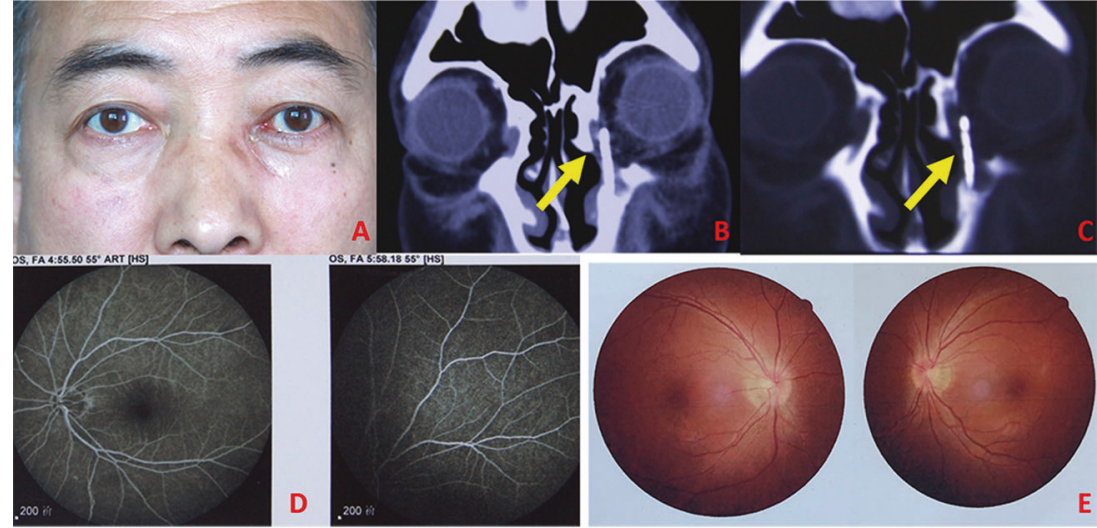

(2) modality, location, and size of the irradiated tumours, as well as the dose of radiation delivered to the eye $[13,15,18-21]$. Unlike $\mathrm{x}$-rays used for external radiotherapy, the I-125 brachytherapy mainly takes the form of $\gamma$-rays, which has lower penetrating power and thus causes less damage to surrounding healthy tissues.

In some unresectable solid tumours like prostate, pancreas, meningioma, I-125 seed was implanted according to three-dimensional radiation therapy planning system (TPS) before operation [22-25]. The tumour is not excised, and the seeds are implanted permanently. In this study, lacrimal sac tumours were removed completely, and the purpose of I-125 seed implantation is to inhibit residual cell recurrence or metastasis. Therefore, we did not use TPS pre-operatively.

Treatment of lacrimal sac tumours depends on the tumour type, malignancy, size, extension and the patient's general health. Ocular treatment is aimed at complete tumour removal, conserving the eye with as much useful vision as possible while eradicating any risk to life. 
Fig. 4 Pre-operative and postoperative images of the patient 4. (1) A firm, localized, nontender mass at the inner canthus a. The picture of the dissected mass b. Gadoliniumenhanced T1-weighted (c, coronal) and T1-plain scan (d, axial) MRI showed midintensity (yellow arrow: the tumour). (2) CT showed that the I-125 seeds were stable and that there was no tumour growth a, b (yellow arrow: the I-125 seeds trips). The fundus photography c results were normal, and vision was 1.0.

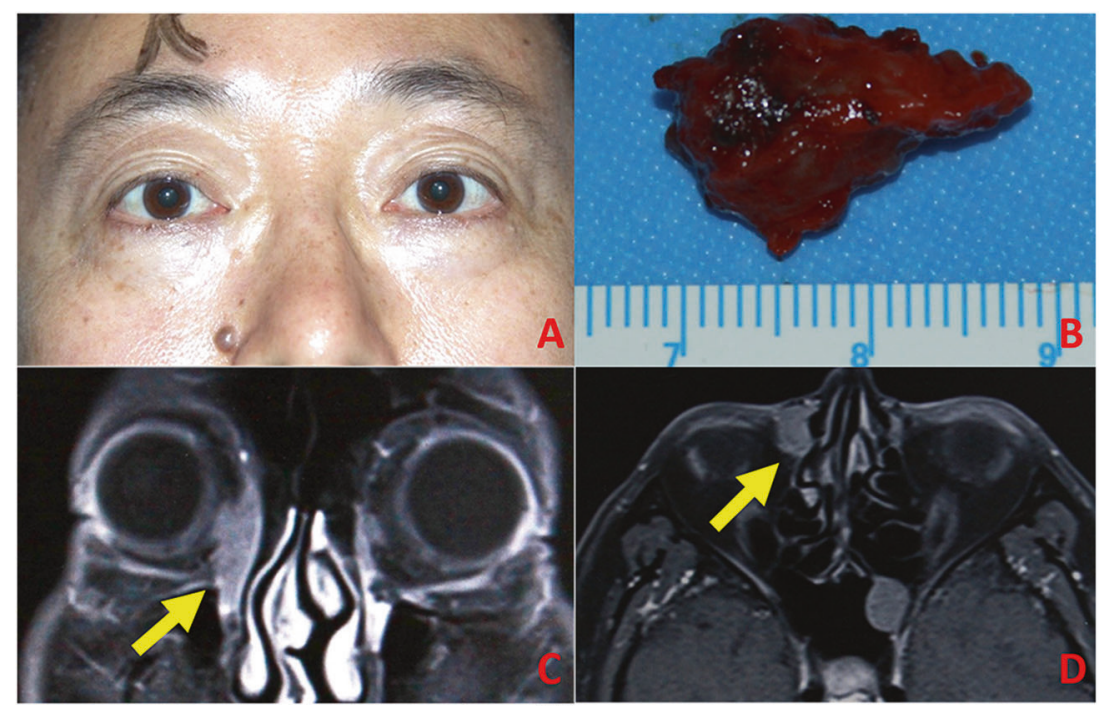

(1)

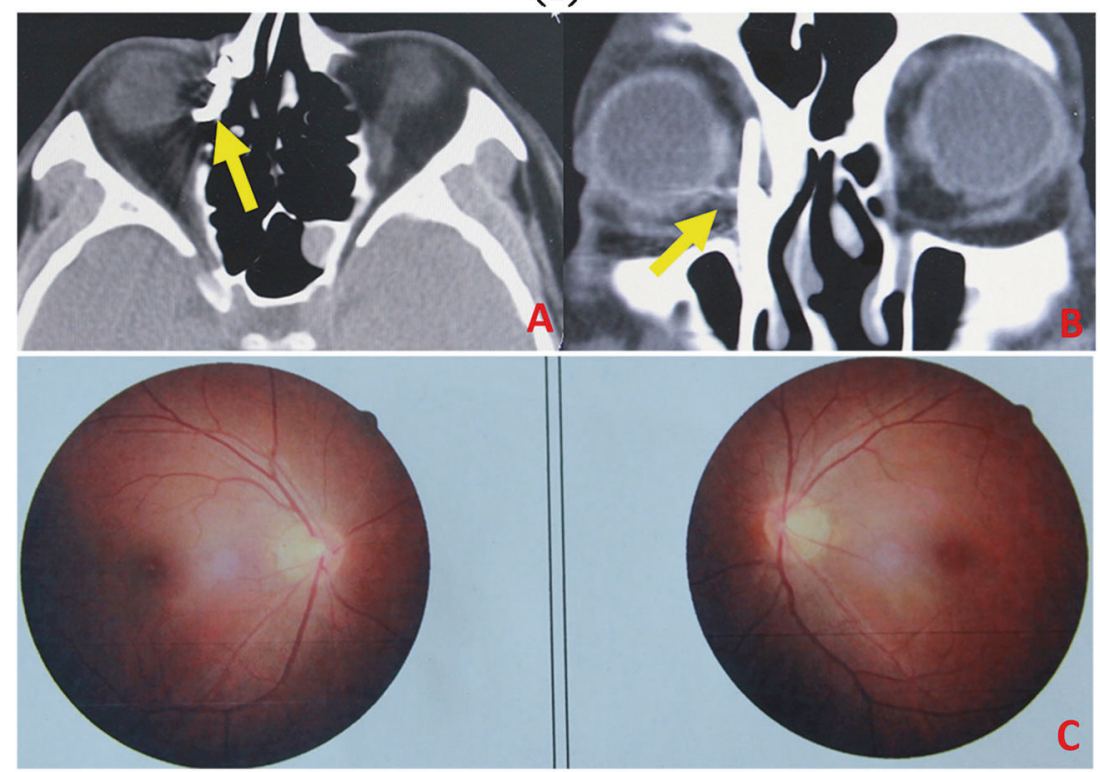

(2)
I-125 seeds are small, and they can be permanently implanted into solid tumours, possibly moving to other areas and inducing serious side effects. They may affect the optic nerve and thus cause vision loss if implanted too deep into the orbit or if they are moved. Therefore, we placed I-125 seeds into the PICC and implanted them into the nasolacrimal canal to ensure that they were stable. After 6 months, the patients could choose whether or not to take them out. All patients did not take out the seeds in this study.

PICC conduits are usually retained in blood vessels for more than one year and experience no obvious rejection. Previous tests showed that implantation of I-125 seeds into PICC pipes did not affect their activity. We implanted seeds into the nasolacrimal duct and subperiosteum without rejection of the surrounding tissues. Thus, these seeds represent a good material.

All patients in this article retained stable visual acuity, which is related to the characteristics of I-125 seeds. I-125 is the most commonly used seed source [26]. The I-125 seed tissue penetration is $1.7 \mathrm{~cm}$. The steeper the dose gradient is, the more concentrated the radiation effect on the basal side of the tumour and, conversely, the less radiation toward the apex. The seeds were implanted far from the eyeball and optic nerve, and there was no radiation effect on the sclera or optic nerve.

Lacrimal sac tumours are often misdiagnosed as chronic dacryocystitis. Some patients were considered to have tumours during DCR, such as the third patient. Many authors advise that an incisional biopsy of the lacrimal sac 
is essential for confirming the diagnosis and may therefore guide adjuvant treatment during DCR [27-29]. In this article, patients 2 and 4 received intraoperative frozen sections, and the tumour was confirmed. Thus, a dacryocystectomy without osteotomy (to avoid the spread of tumour cell into the sinuses) is recommended. Frozen section assessments can confirm the tumour type and help evaluate marginal clearance [1].

I-125 has a half-life of 59 days and is inactive after 6 months. Our patient did not receive any other treatment (chemotherapy or external radiotherapy) after interstitial brachytherapy. The visual acuity in our four treated patients remained stable, with no measurable change from the preoperative acuity. No patient had radiation cataracts, retinopathy papillopathy, or other serious complications, except the third patient with slight radiation dermatitis. The MRI/ CT showed no recurrence of the tumour. Therefore, we conclude that interstitial brachytherapy can inhibit the growth or recurrence of lacrimal sac tumours after surgery.

Patients were selected for interstitial brachytherapy if they had a lacrimal sac malignancy with microscopic evidence of a residual tumour after initial surgical resection. To our knowledge, there have been no prior reports of orbital radiotherapy using this reported technique. There have also been no reports regarding the use of interstitial brachytherapy in lacrimal sac tumours. In this article, we provide an update on our current experience with I-125 interstitial brachytherapy after surgical resection for malignant lacrimal sac tumours.

The implantation technique reported in this article provides a detailed approach to an innovative method of interstitial brachytherapy of extraconal lacrimal sac tumours and ensures the correct implant location.

The only limitation in this study was that the follow-up time was relatively short, mainly because the study was completely new. In the future we will further strengthen follow-up of patients and add additional cases.

\section{Conclusions}

Primary lacrimal sac tumours are extremely rare and require long-term follow-up to assess recurrence and metastasis. Hence, we believe that orbital interstitial brachytherapy represents a valid option in the treatment of malignant lacrimal sac tumours. I-125 interstitial brachytherapy is a reasonable alternative to wide excision or exenteration in malignant lacrimal sac tumours, providing excellent local control and reasonable maintenance of vision. We assessed few patients in our report; thus, we need a larger number of patients and follow-time to confirm the effectiveness of interstitial brachytherapy.

\section{Summary}

\section{What was known before}

- The lacrimal sac was excised completely.

- All I-125 seeds were implanted into the nasolacrimal canal directly.

- There were no recurrence of the tumour and no complications after interstitial brachytherapy.

\section{What this study adds}

- Iodine-125 interstitial brachytherapy can be used as an alternative to wide excision or exenteration of these tumours.

- There was good local control, reasonable maintenance of vision, and good cosmesis.

Funding This study was supported by the National Natural Science Foundation of China (81800808) and the Natural Science Foundation of Shaanxi Province (2016JM8012). None of these funding sources had any role in writing the manuscript or the decision to submit for publication Family Trust.

Author contributions The eight authors are justifiably credited with authorship, according to the authorship criteria. In detail: Conception, design, analysis and interpretation of data, drafting of the manuscript, final approval given; acquisition of data, analysis and interpretation of data, final approval given; and critical revision of manuscript, final approval given.

\section{Compliance with ethical standards}

Conflict of interest The authors declare that they have no conflict of interest.

Informed consent Patient consent was obtained.

Publisher's note Springer Nature remains neutral with regard to jurisdictional claims in published maps and institutional affiliations.

\section{References}

1. Krishna Y, Coupland SE. Lacrimal sac tumors-a review. Asia Pac J Ophthalmol. 2017;6:173-8.

2. Parmar DN, Rose GE. Management of lacrimal sac tumours. Eye. 2003;17:599-606.

3. Shields JA, Shields CL, Freire JE, Brady LW, Komarnicky L. Plaque radiotherapy for selected orbital malignancies: preliminary observations: the 2002 Montgomery Lecture, part 2. Ophthalmic Plast Reconstr Surg. 2003;19:91-5.

4. Klufas MA, Wolden SL, Bohle GC, Wexler LH, Abramson DH. Exenteration and custom implant brachytherapy as a treatment for recurrent primary extraskeletal orbital ewing sarcoma. Ophthalmic Plast Reconstr Surg. 2015;31:e89-91. 
5. Moore RF. Choroidal sarcoma treated by the intraocular insertion of radon seeds. Br J Ophthalmol. 1930;14:145-52.

6. Spraul CW, Lim JI, Lambert SR, Grossniklaus HE. Retinoblastoma recurrence after iodine 125 plaque application. Retina. 1996;16:135-8.

7. Stannard C, Sauerwein W, Maree G, Lecuona K. Radiotherapy for ocular tumours. Eye. 2013;27:119-27.

8. Sealy R, le Roux PL, Rapley F, Hering E, Shackleton D, Sevel D. The treatment of ophthalmic tumours with low-energy sources. Br J Radiol. 1976;49:551-4.

9. Abramson DH, Fass D, McCormick B, Servodidio CA, Piro JD, Anderson LL. Implant brachytherapy: a novel treatment for recurrent orbital rhabdomyosarcoma. J AAPOS. 1997;1:154-7.

10. Bacskulin A, Ehrhardt M, Strietzel M, Pau HW, von Schwanewede $\mathrm{H}$, Guthoff $\mathrm{R}$. An adjuvant afterloading brachytherapy device for use after orbital exenteration in patients with orbital malignancies. Ger J Ophthalmol. 1996;5:484-8.

11. Kim TH, Gerbi BJ, Deibel FC, Priest JR, Khan FM. An afterloading brachytherapy device utilizing thermoplastic material. Radiother Oncol. 1989;15:341-4.

12. Amoaku WM, Archer DB. Fluorescein angiographic features, natural course and treatment of radiation retinopathy. Eye. 1990;4 (Part 5):657-67.

13. Brown GC, Shields JA, Sanborn G, Augsburger JJ, Savino PJ, Schatz NJ. Radiation retinopathy. Ophthalmology. 1982;89:1494-501.

14. Grange JD. Radiation-induced retinopathy. J Fr Ophtalmol. 2001; 24:993-1003.

15. Krema H, Xu W, Payne D, Vasquez LM, Pavlin CJ, Simpson R. Factors predictive of radiation retinopathy post (125)Iodine brachytherapy for uveal melanoma. Can J Ophthalmol. 2011;46:158-63.

16. Lumbroso L, Dendale R, Fourquet A, Desjardins L. Radiationinduced retinopathy. Cancer Radiother. 2002;6:289-95.

17. Zamber RW, Kinyoun JL. Radiation retinopathy. West J Med. 1992; 157:530-3.

18. Finger PT. Tumour location affects the incidence of cataract and retinopathy after ophthalmic plaque radiation therapy. Br J Ophthalmol. 2000;84:1068-70.

19. Pogrzebielski A, Starzycka M, Romanowska-Dixon B, Jakubowska B, Szpakowicz U. The analysis of I125 brachytherapy complications in cases of uveal melanoma. Klin Ocz. 2005; 107:49-53.

20. Puusaari I, Heikkonen J, Kivela T. Ocular complications after iodine brachytherapy for large uveal melanomas. Ophthalmology. 2004;111:1768-77.

21. Summanen $P$, Immonen I, Kivela T, Tommila P, Heikkonen J, Tarkkanen A. Radiation related complications after ruthenium plaque radiotherapy of uveal melanoma. Br J Ophthalmol. 1996; 80:732-9.

22. Bice WS, Prestidge BR, Prete JJ, Dubois DF. Clinical impact of implementing the recommendations of AAPM Task Group 43 on permanent prostate brachytherapy using 125I. American Association of Physicists in Medicine. Int J Radiat Oncol Biol Phys. 1998;40:1237-41.

23. Kumar PP, Good RR, Patil AA, Leibrock LG. Permanent highactivity iodine-125 in the management of petroclival meningiomas: case reports. Neurosurgery. 1989;25:436-41. Discussion 441-442.

24. Marcu L, Quach K. The role of post-implant dosimetry in the quality assessment of prostate implants. The RAH experience. Australas Phys Eng Sci Med. 2006;29:310-4.

25. Peretz T, Nori D, Hilaris B, Manolatos S, Linares L, Harrison L, et al. Treatment of primary unresectable carcinoma of the pancreas with I-125 implantation. Int J Radiat Oncol Biol Phys. 1989;17: 931-5.

26. Kubo H. Determination of the half-life of I-125 encapsulated clinical seeds using a $\mathrm{Si}(\mathrm{Li})$ detector. Med Phys. 1983;10: 889-91.

27. Koturovic Z, Knezevic M, Rasic DM. Clinical significance of routine lacrimal sac biopsy during dacryocystorhinostomy: a comprehensive review of literature. Bosn J Basic Med Sci. 2017;17:1-8.

28. Montalban A, Lietin B, Louvrier C, Russier M, Kemeny JL, Mom $\mathrm{T}$, et al. Malignant lacrimal sac tumors. Eur Ann Otorhinolaryngol Head Neck Dis. 2010;127:165-72.

29. Skinner HD, Garden AS, Rosenthal DI, Ang KK, Morrison WH, Esmaeli B, et al. Outcomes of malignant tumors of the lacrimal apparatus: the University of Texas MD Anderson Cancer Center experience. Cancer. 2011;117:2801-10. 